\section{Muscle belly union associated with simultaneous medial rectus recession for treatment of myopic myopathy: results in 33 eyes}

M Fresina, A Finzi, P Versura and EC Campos

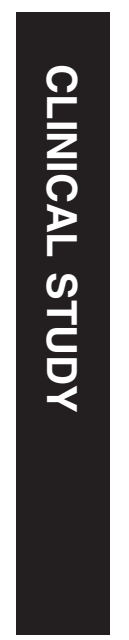

myopathy, when a downward displacement of LR muscle is shown on MRI with coronal sections.

Eye (2014) 28, 557-561; doi:10.1038/eye.2014.15; published online 14 February 2014

Keywords: muscle belly union; medial rectus recession; myopic myopathy; strabismus fixus; myopia

\section{Introduction}

Myopic myopathy is a form of strabismus occurring slowly and progressively in patients with high degenerative myopia. ${ }^{1}$ At first, the term 'heavy eye' was introduced for this form of strabismus with reference to the fact that the myopic eyeball-abnormally grown and consequently heavier-rested on the orbit. ${ }^{2}$ The clinical picture is characterized by hypotropia and esotropia, with limited abduction and elevation. ${ }^{3-5}$ In scientific literature, several pathogenetic hypotheses have been proposed for strabismus occurring in a context of degenerative myopia. They range from an inflammatory disease, which causes an atrophy of the lateral recti muscles, to a mechancal lesion of these muscles due to a contact of the enlarged and deformed eyeball with the orbital wall. ${ }^{6-12}$ Currently, the most accepted theory, first proposed by Krzizok, ${ }^{13}$ suggests that a progressive prolapse of the posterior portion of the eyeball to the superotemporal quadrant of the orbit, outside of the tissues with support function (pulley) and the muscular cone, occurs in patients with high myopia. The prolapsed portion of the bulb causes a nasal displacement of lateral rectus (LR) muscle toward the bottom
Department of Specialist, Diagnostics and Experimental Medicine (DIMES), Ophthalmology Service, University of Bologna, Bologna, Italy

Correspondence: M Fresina, Department of Specialist, Diagnostics and Experimental Medicine (DIMES), Ophthalmology Service, University of Bologna, Via Palagi, 9, 40138 Bologna, Italy. Tel: +3905134 1450; Fax: +39051342821. E-mail: michela.fresina@ libero.it

Received: 15 February 2013 Accepted in revised form: 11 December 2013 Published online: 14 February 2014 
and superior rectus (SR) muscle nasally. This displacement is responsible for a reduction of both abduction and supraduction movements, and an increase in infraduction and adduction, thus resulting in an esodeviation, a hypodeviation and a possible exciclotorsion that are more or less evident depending on the extent of the prolapse. The severity of the clinical picture is highly variable, as it can vary from a smallangle esotropia with a slightly limited abduction, to rarer fixed strabismus. ${ }^{14,15}$ All clinical variants show high axial length of the bulb and alteration of ocular motility toward a restrictive pattern. The treatment of highly myopic hypo-esotropia is essentially surgical. Several traditional surgical techniques have been used over the years. ${ }^{15-17}$ These procedures are effective in some cases, but esotropia often reappears after a few months. ${ }^{14,15,18}$ Muscle belly union was introduced by Yokoyama in $2001^{19}$ and has become a viable alternative to treat this condition. A retroequatorial approach is performed by a bridge suture of the intact belly of the LR muscle and SR muscle-or their superior and temporal portions, respectively-obtained with an incision. Therefore, the intervention allows to bring the elongated myopic bulb back to its original position inside the muscular cone. Several reports have supported the effectiveness of this surgery, although the technique remains controversial for the correction of smaller-angle deviations. ${ }^{20-26}$

\section{Materials and methods}

This is a retrospective, nonrandomized study performed on 33 eyes of 26 patients (19 female and 7 male) who underwent muscle belly union to treat myopic myopathy and strabismus fixus from January 2004 to October 2012. Their mean age was $48.3 \pm 15.8$ years, going from 18 to 75 years. The inclusion criterion for this study was acquired restrictive strabismus with axial high myopia: 22 patients had eso-hypotropia (15 unilateral and 7 bilateral), and 4 patients had unilateral strabismus fixus. Patients who had previously undergone strabismus surgery were not included in the study. The following items were recorded preoperatively and postoperatively: best-corrected visual acuity (BCVA), refraction, intraocular pressure (IOP), complete orthoptic assessment, including measurement of the angle of deviation and maximal abduction. Early Treatment Diabetic Retinopathy Study (ETDRS) charts were used to test BCVA at a distance of $4 \mathrm{~m}$. Patients who failed to read the largest letters $4 \mathrm{~m}$ away were tested at a distance of $1 \mathrm{~m}$. Lighting conditions were standardized, using ETDRS Eye Charts No. 2121 and ETDRS Illuminator Cabinet s/n 106 (Precision Vision, La Salle, IL, USA). BCVA was scored as the total number of letters read correctly and that number was transformed into minimum angle of resolution (logMAR) units logarithm. Patients who failed to read any letters were tested using counting fingers $(\mathrm{CF})$, hand movements $(\mathrm{HM})$, and light perception (P). For BCVA less than CF $0.5 \mathrm{~m}$, arbitrary logMAR values of 2.2 (CF in front of the eye), 2.3 (HM), $2.5(\mathrm{P})$, and 3 (no light perception) were used. Ideally, measurements of the angle of deviation were obtained using prism bars and alternating cover test at 5 and $0.33 \mathrm{~m}$; however, when this proved impossible, Krimsky's test was used. A Goldmann perimeter was used to measure the maximal abduction, that is, the excursion expressed in degrees the eye is able to perform from the primary position to the position of maximum abduction. Pictures of the eyes in all gaze directions were taken before and after the surgical treatment (Figures 1 and 2). Magnetic resonance imaging (MRI) of the orbits was performed preoperatively in all patients to exclude other possible causes of restrictive strabismus and to evaluate the displacement of extraocular muscles. As suggested by Yokoyama, ${ }^{26}$ we used coronal section of the orbits-obtained by MRI-to analyze the degree of dislocation of the bulb, about $9 \mathrm{~mm}$ in front of the junction with the optic nerve, to avoid the possible influence of globe shape in the event that, for example, there were a myopic staphyloma. We used AutoCAD software (Autodesk, San Rafael, CA, USA) to quantitatively investigate the relationships between the

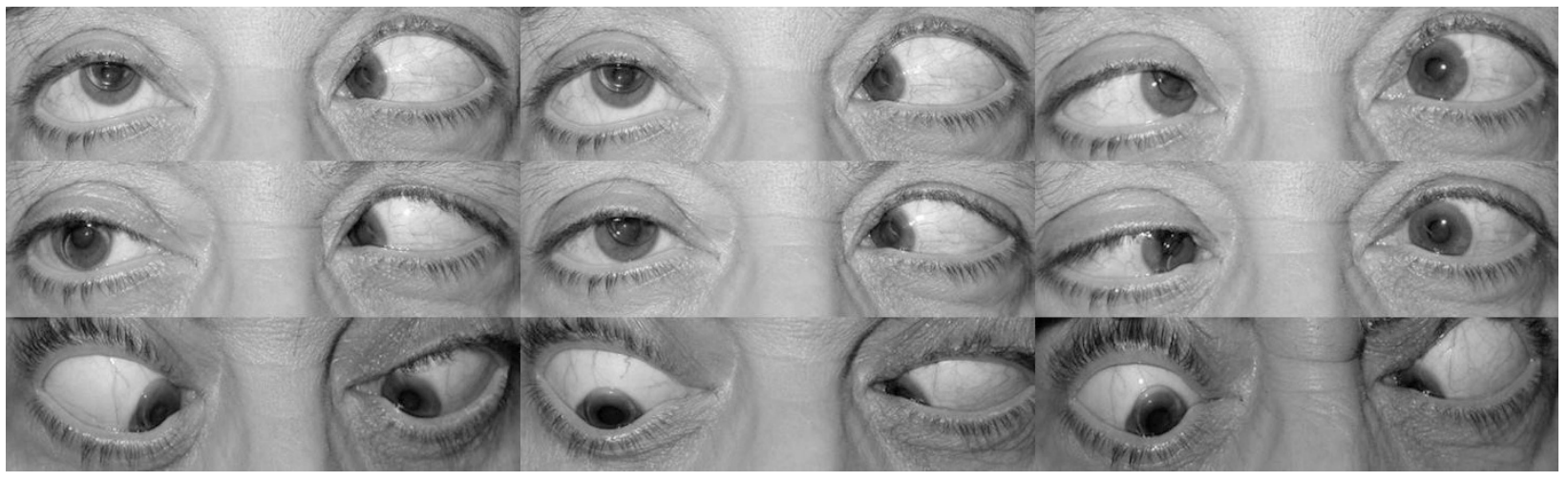

Figure 1 Preoperative pictures of the eyes in all gaze directions in patient with bilateral myopic myopathy. 
different structures. The angle subtended between two lines connecting the center of the orbit $(G)$ and the center of the LR and SR bellies was calculated (Figure 3). When the angle is $\sim 180^{\circ}$ or more, more than half of the globe cross-section is located outside the muscle cone through an opening between SR and LR muscles. All patients with this feature underwent surgery according to the procedure described by Yokoyama in association with recession of the ipsiLR muscle.

Surgery was performed under general anesthesia and all patients signed an informed consent before the surgery. All examinations and surgical procedures were conform to the tenets of the Declaration of Helsinki.

LR and SR muscles were approached using a limbal peritomy and isolated separately. Approximately half of each muscle belly was sutured together without muscle splitting and using a 4.0 no-absorbable mersilene suture, $\sim 12-15 \mathrm{~mm}$ posterior to the muscular insertion, without scleral fixation. The intraoperative motility was evaluated by means of forced duction test before and after muscle belly union to assess whether a medial rectus recession was required to correct residual restriction in abduction. The force duction test was positive in all 33 eyes so a recession of medial rectus muscle, dosed on the basis of the angle of esotropia measured preoperatively (ranged $5.0-12.0 \mathrm{~mm}$ ), was always performed. SPSS Statistics 12.0 software (SPSS Inc., Chicago, IL, USA) was used for statistical analysis. Comparisons were recorded as $95 \%$ confidence intervals, using a paired sample $t$-test.

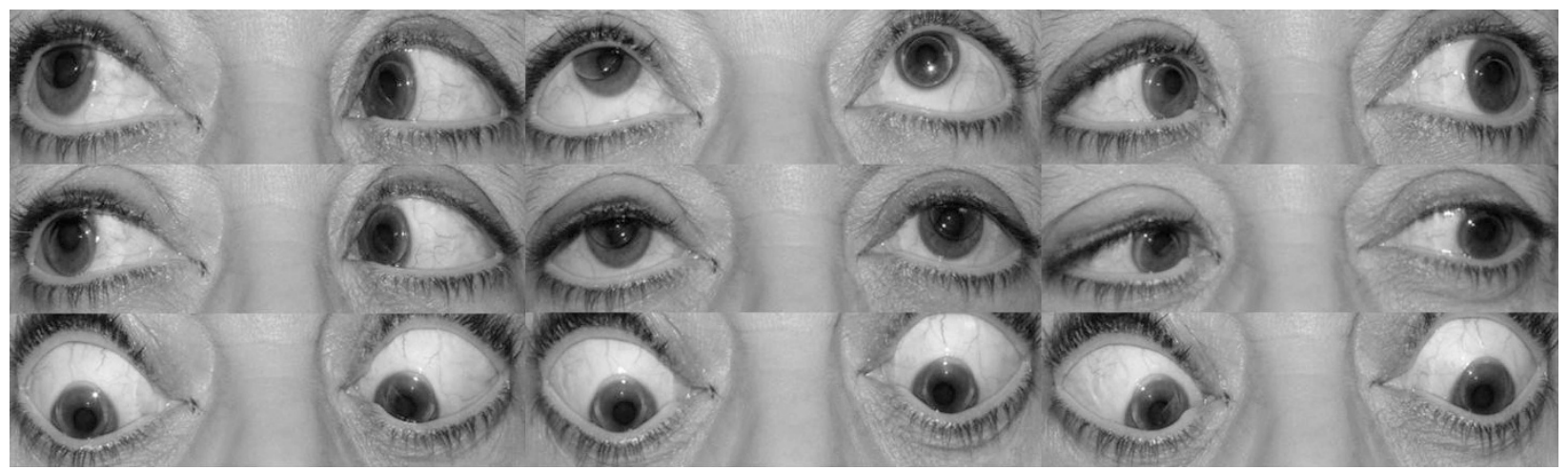

Figure 2 Postoperative pictures of the eyes in all gaze directions in patient who underwent bilateral Yokoyama's surgical procedure.

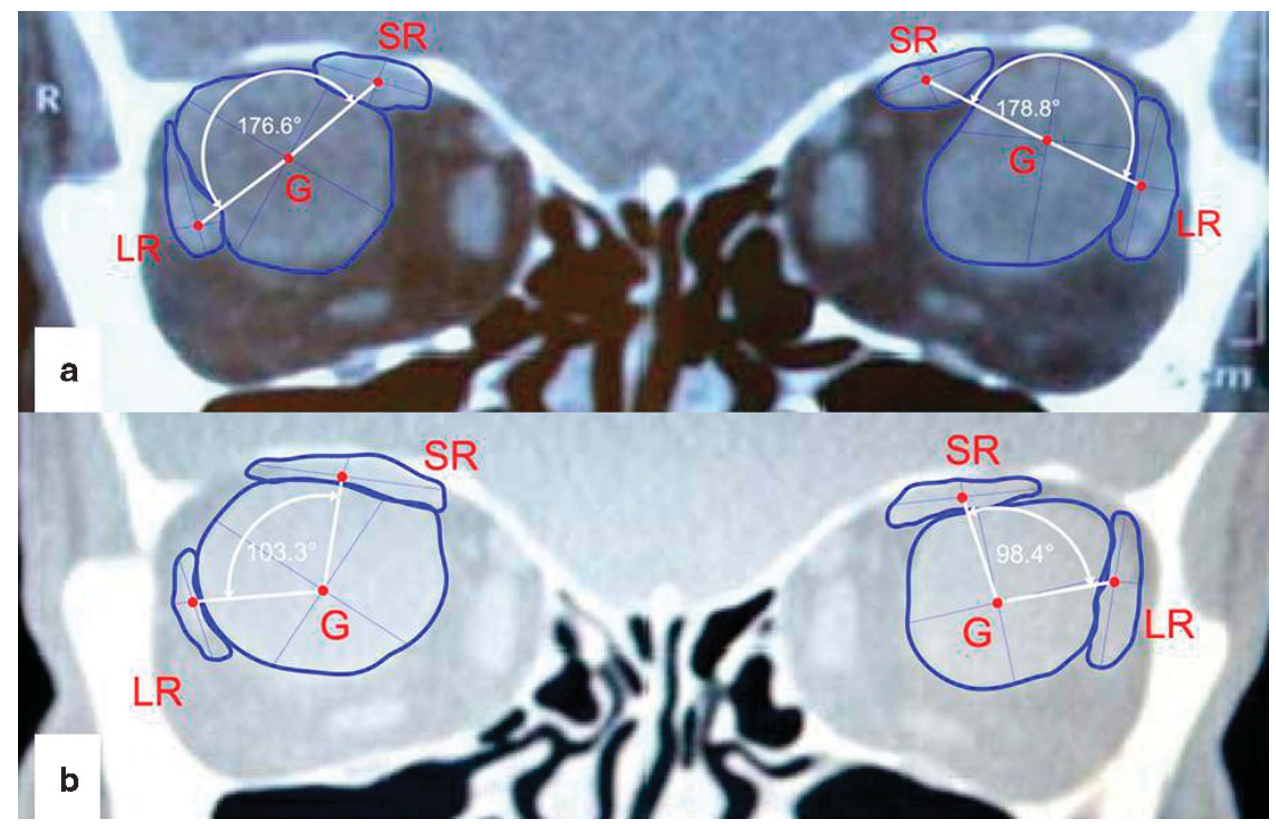

Figure 3 Preoperative (a) and postoperative (b) coronal MRI section of patient who underwent bilateral Yokoyama's surgical procedure. 


\section{Results}

The mean BVCA before surgery was $0.97 \pm 0.96 \log$ MAR (range 0.1-3 logMAR) and did not change at postoperative controls. High myopia was defined as an axial length of $\geq 27.0 \mathrm{~mm}$ or as refractive values $>-6$ diopters (D). The mean spherical equivalent was $-22.9 \pm 5.6 \mathrm{D}$. The preoperative mean hypotropia and esotropia were, respectively, $10.2 \pm 3.9$ prism diopter (PD) and 46.2 \pm 15.5 PD. Six months after surgery, the mean hypotropia was $2.48 \pm 2.00 \mathrm{PD}(P<0.001)$ and the mean esotropia was $7.36 \pm 9.09$ PD $(P<0.001)$. There was also a statistical increment of the mean maximal abduction, going from $14 \pm 7$ to $26 \pm 4$ degrees $(P<0.001)$ (Table 1$)$. No over-correction od adduction limitations were present after surgery. Before surgery, 12 patients presented with incoercible diplopia, whereas after it only 6 patients reported mild diplopia resolved with the use of prismatic lenses (from 2 to $10 \mathrm{PD}$ ). The mean IOP before surgery was $12 \pm 4 \mathrm{~mm} \mathrm{Hg}$ and there was no significant change at postoperative controls, except in three cases.

Two surgical complications were recorded: three cases of rise in IOP, solved after 3 months with topical hypotonic therapy (dorzolamide hydrochloride $2 \%+$ timolol maleate $0.5 \%, 2$ times per day), and a case of nasal corneal dellen, solved after 15 days with topical antibiotic, anti-inflammatory therapy and nocturnal occlusion.

\section{Discussion}

Different approaches to correct myopic myopathy cases have been tried, and the degrees of success were varied. ${ }^{15-19}$ In general, the standard recession-resection procedures are ineffective over time, and LR resection may augment the muscle displacement. Yokoyama's surgical procedure ${ }^{19}$ restores the dislocated globe back into the muscle cone by a bridge suture of the LR and SR muscles bellies, or their superior and temporal portions, respectively. It represents an effective procedure for highly myopic strabismus and strabismus fixus in high myopia. We always associated the recession of the medial rectus muscle, because a restriction in abduction was still present during the intraoperative forced duction test.

Table 1 Surgical results in patients with highly myopic strabismus

\begin{tabular}{lccc}
\hline & $\begin{array}{c}\text { Vertical deviation } \\
\text { (hypotropia) }\end{array}$ & $\begin{array}{c}\text { Horizontal } \\
\text { deviation } \\
\text { (esotropia) }\end{array}$ & $\begin{array}{c}\text { Maximal } \\
\text { abduction }\end{array}$ \\
\hline Preoperative & $10.2 \pm 3.9 \mathrm{PD}$ & $46.2 \pm 15.5 \mathrm{PD}$ & $14 \pm 7^{\circ}$ \\
Postoperative & $2.48 \pm 2.00 \mathrm{PD}^{\mathrm{a}}$ & $7.36 \pm 9.09 \mathrm{PD}^{\mathrm{a}}$ & $26 \pm 4^{\circ}$ \\
\hline
\end{tabular}

Values are presented as mean $( \pm)$, standard deviation (degrees).

${ }^{\text {a }} P<0.001$ if compared with preoperative period.
This surgical technique-without scleral fixationgreatly reduces the risk of scleral perforation, making the procedure safe and simple to learn and perform. The use of non-absorbable sutures ensures that the muscles retain their position over time.

During a 6-month follow-up no muscle slips were recorded. Only four patients presented postoperative complications, which were quickly resolved without permanent sequelae. The symptom of diplopia was successfully resolved with surgery. The mean maximal abduction was significantly increased in all patients. No patient experienced a decrease in preoperative BCVA. The surgery provided psychosocial benefit to all patients.

In our opinion, the intervention proposed by Yokoyama-coupled with the recession of the ipsilateral medial rectus muscle when considered convenient-is the elective surgical technique in highly myopic patients with eso-hypotropia, when a downward displacement of the LR muscle is recorded shown on the MRI, performed with coronal sections.

\section{Summary}

What was known before

- Myopic myopathy is a form of strabismus known for many years for which many pathogenetic theories and numerous surgical approaches have been proposed.

- At the moment, the most effective surgical technique seems to be that proposed by Yokoyama.

- In the literature a limited number of cases of patients operated upon this surgical technique have been described.

What this study adds

- Our study covered a large number of patients with myopic myopathy operated with the surgical technique proposed by Yokoyama.

- In these patients we always associate the recession of the medial rectus muscle of the affected eye/eyes to obtain the best anatomical and functional results.

\section{Conflict of interest}

The authors declare no conflict of interest.

\section{Acknowledgements}

This work was partially supported by a grant from Fondazione Cassa di Risparmio di Bologna and RFO (ex-60\%) fundus from University of Bologna, all to ECC.

\section{Disclosure}

The authors have full control of all primary data and they agree to allow Eye review their data if requested. 


\section{References}

1 Noorden GK von, Campos EC. Binocular Vision and Ocular Motility. 6th ed. Mosby Inc.: St Louis, 2002.

2 Bagshaw J. The heavy eye phenomenon: a preliminary report. Br J Ophthalmol 1966; 23: 73-78.

3 Taylor R, Whale K, Raines M. The heavy eye phenomenon: orthoptic and ophthalmic characteristics. Ger J Ophthalmol 1995; 4: 252-255.

4 Kaynak S, Durak I, Ozaksoy D, Canda T. Restrictive myopic myopathy: computed tomography, magnetic resonance imaging, echography, and histological findings. Br J Ophthalmol 1994; 78: 414-415.

5 Mansour AM, Wang F, El-Baba F, Henkind P. Ocular complications in strabismus fixus convergens. Ophthalmologica 1987; 195: 161-166.

6 Hugonnier R, Magnard P. Syndrome nerveux de la myopie forte. Bull Mem Soc Fr Ophthalmol 1960; 73: 80.

7 Hugonnier R, Magnard P. Oculomotor disequilibrium observed in cases of severe myopia. Ann Ocul 1969; 202: 713-724.

8 Demer JL, Noorden GK von. High myopia as an unusual cause of restrictive motility disturbance. Surv Ophthalmol 1989; 33: 281-284.

9 Bagolini B, Tamburrelli C, Dickmann A, Colosimo C. Convergent strabismus fixus in high myopic patients. Doc Ophthalmol 1990; 74: 309-320.

10 Lanthony P. The heavy eye syndrome. Bull Mem Soc Fr Ophtalmol 1985; 96: 26-28.

11 Herzau V, Ioannakis K. Pathogenesis of eso- and hypotropia in high myopia. Klin Monbl Augenheilkd 1996; 208: 33-36.

12 Yokoyama T, Tabuchi H, Ataka S, Shiraki K, Maki T, Mochizuchi K. The mechanism of development in progressive esotropia with high myopia. In de Faber JT (ed) Transactions of the 26th ESA meeting. Swets \& Zeitlinger: Lisse (Netherlands), 2000, pp 218-221.

13 Krzizok TH, Schroeder BU. Measurement of recti eye muscles paths by magnetic resonance imaging in highly myopic and normal subjects. Invest Ophthalmol Vis Sci 1999; 40: 2554-2560.
14 Sturm V, Menke MN, Chaloupka K, Landau K. Surgical treatment of myopic strabismus fixus: a graded approach. Graefes Arch Clin Exp Ophthalmol 2008; 246 1323-1329.

15 Hayashi T, Iwashige H, Maruo T. Clinical features and surgery for acquired progressive esotropia associated with severe myopia. Acta Ophthalmol Scand 1999; 77: 66-71.

16 Webb H, Lee J. Acquired distance esotropia associated with myopia. Strabismus 2004; 12: 149-155.

17 Nishida Y, Hayashi O, Oda S, Kakinoki M, Miyake T, Inoki $\mathrm{Y}$ et al. A simple muscle transposition procedure for abducens palsy without tenotomy or splitting muscles. Jpn J Ophthalmol 2005; 49: 179-180.

18 Nakagawa S, Kii T, Suzuki J, Takeda M, Nakagawa T, Sato Y et al. 5 cases of strabismus fixus. Nippon Ganka Kiyo 1989; 40: 656-662.

19 Yokoyama T, Ataka S, Tabuchi H, Shiraki K, Miki T. Treatment of progressive esotropia caused by high myopia-a new surgical procedure based on its pathogenesis. In de Faber JT (ed) Transactions of the 27th ESA Meeting. Swets \& Zeitlinger: Lisse (Netherlands), 2002, pp 145-148.

20 Wong I, Leo SW, Khoo BK. Loop myopexy for treatment of myopic strabismus fixus. J AAPOS 2005; 9: 589-591.

21 Durnian JM, Maddula S, Marsh IB. Treatment of 'heavy eye syndrome' using simple loop myopexy. J AAPOS 2010; 14: 39-41.

22 Rowe FJ, Noonan CP. Surgical treatment for progressive esotropia in the setting of high myopia. J AAPOS 2006; 10: 596-597.

23 Leo SW, Del Monte MA. Surgical correction of myopic strabismus fixus by modified loop transposition with scleral myopexy. J AAPOS 2007; 11: 95.

24 Ahadzadeghan I, Akbary MR, Ameri A, Anvari F, Jafari AK, Rajabi MT. Muscle belly union for treatment of myopic strabismus fixus. Strabismus 2009; 17: 57-62.

25 Godeiro KD, Kirsch D, Tabuse MK, Cronemberger M. Yamada's surgery for treatment of myopic strabismus fixus. Int Ophthalmol 2009; 29: 305-308.

26 Yamaguchi M, Yokoyama T, Shiraki K. Surgical procedure for correcting globe dislocation in highly myopic strabismus. Am J Ophthalmol 2010; 149: 341-346. 